\title{
ACTIVIDAD ENZIMÁTICA Y CAPACIDAD ANTIOXIDANTE EN MENTA (Mentha piperita L.) ALMACENADA BAJO REFRIGERACIÓN1
}

\author{
María Teresa Martínez-Damián², Oscar Cruz-Álvarez², María Teresa Beryl Colinas-León², \\ Juan Enrique Rodríguez-Pérez $z^{2}$ Sweetia Paulina Ramírez-Ramírez²
}

\section{RESUMEN}

Actividad enzimática y capacidad antioxidante en menta (Mentha piperita L.) almacenada bajo refrigeración. El objetivo de esta investigación fue estudiar el efecto de las bajas temperaturas sobre la actividad enzimática y capacidad antioxidante en menta (Mentha piperita L.). Se cuantificó su comportamiento en almacenamiento a 6 y $10^{\circ} \mathrm{C}$ con respecto a un testigo (temperatura ambiente), para esto, se condujo un experimento en laboratorio durante el ciclo primavera-verano de 2011 en la Universidad Autónoma Chapingo, Texcoco, México. Se evaluó la actividad enzimática de peroxidasa (POD), polifenol oxidasa (PFO), superóxido dismutasa (SOD) y catalasa (CAT), de igual forma se determinó la capacidad antioxidante (CA), contenido de fenoles totales (FT) y de vitamina C (VC). La temperatura de $10^{\circ} \mathrm{C}$ incrementó la actividad enzimática de POD de 194,72 a 306,29 U/g de PF; así como la actividad antioxidante de 262,57 a 327,32 mm TEAC/g de PF. Por otra parte, a 6 y $10^{\circ} \mathrm{C}$ aumentaron los contenidos de FT y VC, inverso a lo sucedido con la actividad de SOD que disminuyó de 53,68 a 26,22 U/g de PF. El tratamiento a temperatura ambiente favoreció una mayor actividad de PFO (90,02 U/g de PF). La refrigeración incrementó la actividad enzimática de POD, el contenido de FT y VC. Por otro lado, la actividad de SOD disminuyó y la CA fue mayormente afectada por la temperatura de almacenamiento a $10^{\circ} \mathrm{C}$.

Palabras clave: peroxidasa, polifenol oxidasa, superóxido dismutasa, catalasa, ácido ascórbico.

\begin{abstract}
Enzymatic activity and antioxidant capacity in mint (Mentha piperita L.) under refrigerated storage. The objective of this investigation was to study the effect of low temperatures over the enzymatic activity and antioxidant capacity in mint (Mentha piperita L.), behavior was quantified during storage at 6 and $10^{\circ} \mathrm{C}$ with respect to a control treatment (at room temperature), for this purpose, a laboratory experiment was conducted during a spring-summer cycle of 2011 at the University Autonomous of Chapingo, Texcoco, Mexico. The activity of enzymes, such as peroxidase (POD), polyphenol oxidase (PPO), superoxide dismutase (SOD) and catalase (CAT), was determined; as well as the antioxidant capacity (AC), total phenol (TP) and vitamin C (VC) content. The main result found is that the enzymatic activity of POD increased from 194,72 to $306.29 \mathrm{U} / \mathrm{g}$ of $\mathrm{FW}$ (fresh weight) at $10^{\circ} \mathrm{C}$; the antioxidant activity also increased from 262,57 to $327,32 \mathrm{~mm} \mathrm{TEAC/g}$ of $\mathrm{WF}$ at the same temperature. On the other hand, at $6^{\circ} \mathrm{C}$ and $10^{\circ} \mathrm{C}$ the contents of $\mathrm{WF}$ and $\mathrm{VC}$ increased, but the effect on the activity of SOD was inverse as it decreased from 53,68 to $26,22 \mathrm{U} / \mathrm{g}$ of WF. The treatment room temperature contributes to an increased activity of the PPO (90,02 U/g of WF). The use of refrigeration increased enzymatic activity of POD, PT contents and VC. Also it was found that the SOD activities decreased and the AC was greatly affected at a $10^{\circ} \mathrm{C}$ storage temperature.
\end{abstract}

Key words: peroxidase, polyphenol oxidase, superoxide dismutase, catalase, ascorbic acid.

\footnotetext{
Recibido: 15 de agosto, 2012. Aceptado: 1 de abril, 2013. Este trabajo es parte de la tesis de doctorado en Ciencias en Horticultura, Universidad Autónoma Chapingo, México, del segundo autor.

2 Universidad Autónoma Chapingo. Departamento de Fitotecnia. Km. 38.5 Carr, Fed. Méx.-Tex. Chapingo, Texcoco, Estado de México. C.P. 56230. México. Teléfono: (595) 9521500 ext.6163. teremd13@gmail.com, cruzalvarezoscar@gmail.com, lozcol@gmail.com, erodriguezx@yahoo.com.mx, sweetia.ramirez@gmail.com
} 


\section{INTRODUCCIÓN}

Se ha observado que las funciones principales en el cuerpo humano dependen de las reacciones de oxidación, que a su vez producen especies reactivas de oxígeno (ERO) como producto intermedio del metabolismo (Nickavar et al. 2008), en las que se incluyen al anión radical superóxido, peróxido de hidrógeno y el ión hidroxilo (Rodríguez et al. 2006, Serteser et al. 2009).

Las especies reactivas de oxígeno no solo están fuertemente asociadas con la peroxidación de lípidos y el consecuente deterioro de alimentos, sino que también están involucradas en el desarrollo de enfermedades cancerígenas (Rodríguez et al.2010), por lo que el consumo de alimentos con alto contenido de antioxidantes es importante en la prevención de estas (Vargas et al. 2005, Erdemoglu et al. 2006), debido a que son compuestos que pueden retrasar o inhibir la oxidación de lípidos y otras moléculas mediante la inhibición de la propagación de ERO (Velioglu et al. 1998).

Los sistemas de defensa antioxidantes en las plantas implican por un lado, un sistema no enzimático, donde se promueve la síntesis de numerosos metabolitos secundarios (ácidos fenólicos, flavonoides, taninos, vitaminas, y terpenoides) en la ruta de los fenilpropanoides (Yanishlieva et al. 2006, Karousou et al. 2007); y por el otro, un sistema antioxidante enzimático que se encuentra localizado en varios compartimentos de la célula (Zheng y Wang 2001), en las que se incluye a la superóxido dismutasa (SOD) que convierte al superóxido a $\mathrm{H}_{2} \mathrm{O}_{2}$, peroxidasa (POD), la cual convierte el $\mathrm{H}_{2} \mathrm{O}_{2}$ a agua y catalasa (CAT) que elimina a el $\mathrm{H}_{2} \mathrm{O}_{2}$ (Oueslati et al. 2010).

La búsqueda de antioxidantes naturales, especialmente en plantas, ha aumentado considerablemente, debido a que los sintéticos sufren el inconveniente que son volátiles y se descomponen fácilmente a altas temperaturas (Dorman et al. 2003, Tao et al. 2007); adicionalmente, algunos de ellos han sido suspendidos por causar o promover efectos negativos en la salud (Djeridane et al. 2006), por lo que algunas plantas aromáticas han sido ampliamente estudiadas por su intensa actividad antioxidante (Nickavar et al. 2008), entre las que destacan: albahaca, salvia, orégano y menta (Djeridane et al. 2006, Tepe et al. 2006), lo que ha resultado en el desarrollo de formulaciones de antioxidantes naturales (Miliauskas et al. 2004).

En el caso particular de la menta (Mentha piperita L.), esta es un híbrido entre Mentha aquatica L. y
Mentha viridis L., que pertenece a la familia Lamiaceae conformada por aproximadamente 220 géneros y 3300 especies (Mimica-Dukin y Bozin 2008). De acuerdo con lo descrito en la Farmacopea Europea IV (2002) esta planta es usada para tratar diversos padecimientos gastrointestinales y gastritis, así como en la industria farmacéutica y agroalimentaria (Yanishlieva et al. 2006). También se ha reportado como una fuente importante de compuestos polifenólicos y antioxidantes (Nickavar et al. 2008, Tepe et al. 2006, Neves et al. 2009). Sin embargo, a pesar de la existencia de estos estudios, no se ha relacionado con su comportamiento en almacenamiento a bajas temperaturas, por lo que se plantea la presente investigación con el objetivo de evaluar el efecto poscosecha de tres temperaturas de almacenamiento sobre la actividad enzimática y capacidad antioxidante en menta (Mentha piperita L.).

\section{MATERIALES Y MÉTODOS}

\section{Material vegetal}

Se utilizó como material vegetal a la menta (Mentha piperita L.), variedad "mint moroco" que fue proporcionada por la empresa Glezte SPR de RI, localizada en Axochiapan, Morelos, México; donde fue cosechada y preenfriada por 24 horas a $5^{\circ} \mathrm{C}$ con el propósito de eliminar el calor de campo. El municipio de Axochiapan se ubica geográficamente entre los paralelos $18^{\circ} 30^{\prime}$ de latitud norte y los $98^{\circ} 45^{\prime}$ de longitud oeste, así como a una altitud de $1030 \mathrm{msnm}$ (Anónimo 1988).

\section{Ubicación del experimento}

El trabajo experimental se llevó a cabo durante el ciclo primavera-verano de 2011, en los Laboratorios de Fisiología de Frutales y en el de Usos Múltiples del Departamento de Fitotecnia de la Universidad Autónoma Chapingo, ubicada en Texcoco, Estado de México, México.

\section{Tratamientos evaluados}

El experimento consistió de almacenar menta a tres temperaturas $\left(6,10^{\circ} \mathrm{C}\right.$ y a ambiente (testigo)), para lo cual se pesaron manojos de $250 \mathrm{~g}$ y se colocaron en bolsas de polietileno de baja densidad, 
perforadas manualmente, simulando el proceso de comercialización a Estados Unidos y Canadá.

\section{Diseño experimental}

El diseño experimental fue completamente al azar con cuatro repeticiones. La unidad experimental consistió de una bolsa de polietileno perforada con $250 \mathrm{~g}$ de material vegetal (se incluyen tallos), de la cual se utilizaron $50 \mathrm{~g}$ de menta (solo hojas) para la elaboración de polvo de acetona a partir de la cual se evaluó la actividad enzimática de peroxidasa, polifenoloxidasa, catalasa y superóxido dismutas, así como la capacidad antioxidante y el contenido de fenoles totales; para el contenido de vitamina $\mathrm{C}$ se usaron $5 \mathrm{~g}$ (en fresco). Todas las evaluaciones se llevaron a cabo durante quince días en periodos de tres días.

\section{Reactivo}

\section{Polvo de acetona}

Se realizó de acuerdo con Alia et al. (2002), para lo cual se tomaron $50 \mathrm{~g}$ de hojas, más $100 \mathrm{ml}$ de acetona fría $\left(-15^{\circ} \mathrm{C}\right)$ grado reactivo, se licuó durante un minuto y se filtró al vacío; el proceso se repitió en cuatro ocasiones, se dejó secar a temperatura ambiente y se almacenó en un ultracongelador $\left(-80^{\circ} \mathrm{C}\right)$.

Se tomó el peso del polvo obtenido para determinar la relación peso fresco : peso seco (PF:PS). El filtrado se guardó en refrigeración (entre 2 y $5^{\circ} \mathrm{C}$ ) bajo condiciones de oscuridad total.

\section{Enzimas}

\section{Peroxidasa $(P O D)$}

Se pesaron $0,10 \mathrm{~g}$ de polvo de acetona los cuales se homogeneizaron en frío con $5 \mathrm{ml}$ de Tris- $\mathrm{HCl} 0,1 \mathrm{mM}$ $(7,1)$ conteniendo $1 \%$ de polivinilpirrolidona (PVP), se centrifugó a 12000 x g x $30 \mathrm{~m}$ a $4^{\circ} \mathrm{C}$. El ensayo se realizó de acuerdo a Flurkey y Jen (1978), con las siguientes modificaciones: la mezcla de ensayo tuvo un volumen total de $3 \mathrm{ml}$, para lo cual se mezclaron $2,6 \mathrm{ml}$ de amortiguador Tris- $\mathrm{HCl} 100 \mathrm{Mm}(\mathrm{pH}$ 7,1), $0,25 \mathrm{ml}$ de guayacol $0,1 \mathrm{M}, 0,1 \mathrm{ml}$ de peróxido de hidrógeno $0,25 \%$ y $0,05 \mathrm{ml}$ del sobrenadante. Se evaluó el cambio de absorbancia en 1,5 min a $470 \mathrm{~nm}$. La actividad enzimática se reportó como $\mathrm{U} / \mathrm{g}$ de $\mathrm{PF}$, donde $\mathrm{U}=$ Unidad de actividad enzimática y una unidad es igual a la formación de $1 \mu \mathrm{mol}$ de tetraguaiacol/min. Los ensayos se realizaron a temperatura ambiente.

\section{Polifenol oxidasa (PFO)}

La extracción de PFO es similar a POD. La PFO se evaluó mediante el método propuesto por Laminkara (1995) con modificaciones, la enzima se extrajo a partir de $0,2 \mathrm{~g}$ de polvo de acetona con $5 \mathrm{ml}$ de Tris$\mathrm{HCl}$ frío, $100 \mathrm{mM}(\mathrm{pH} \mathrm{7,1)}$, que se mezclaron con un homogeneizador de tejidos durante $30 \mathrm{~s}$, posteriormente la mezcla se centrifugó por 20 minutos a $12000 \mathrm{x} \mathrm{g}$ a $4^{\circ} \mathrm{C}$, el sobrenadante se utilizó para el ensayo, donde se evaluó el cambio de absorbancia a $420 \mathrm{~nm}$. La mezcla de reacción consistió de $2 \mathrm{ml}$ de catecol $(60 \mathrm{mM})+$ $0,05 \mathrm{ml}$ del sobrenadante (se determinó el cambio de absorbancia en un minuto). Los ensayos se realizaron a temperatura ambiente, entre $20-22^{\circ} \mathrm{C}$. La actividad enzimática se reportó como $\mathrm{U} / \mathrm{g}$ de $\mathrm{PF}$, donde $\mathrm{U}=$ Unidad de actividad enzimática y una unidad es igual a la formación de $1 \mu \mathrm{mol}$ de o-benzoquinona/min. Los ensayos se realizaron a temperatura ambiente.

\section{Superóxido dismutasa (SOD)}

A $0,1 \mathrm{~g}$ de polvo de acetona, se le adicionaron $5 \mathrm{ml}$ de amortiguador de fosfatos frío $(\mathrm{pH} 7,8)$ y se homogeneizaron durante un minuto, la mezcla se centrifugó a $12000 \mathrm{x}$ g por $30 \mathrm{~min}$ a $4^{\circ} \mathrm{C}$. El sobrenadante se utilizó para el ensayo enzimático propuesto por Beyer y Fridovich (1987), donde se propone la siguiente mezcla de reacción; $27 \mathrm{ml}$ de amortiguador de fosfatos $0,05 \mathrm{M}$ (pH 7,8), conteniendo 0,1 mM de EDTA, 1,5 $\mathrm{ml}$ de L-metionina $(30 \mathrm{mg} / \mathrm{ml}), 1 \mathrm{ml}$ de Nitro Blue Tetrazolium $(1,41 \mathrm{mg} / \mathrm{ml})$ y $0,75 \mathrm{ml}$ de Triton X-100 al $1 \%$. A $3 \mathrm{ml}$ de esta mezcla se adicionaron $0,03 \mathrm{ml}$ de riboflavina $(4,4 \mathrm{mg} / \mathrm{ml})$ y $0,4 \mathrm{ml}$ de sobrenadante, todo esto bajo oscuridad total, posteriormente esta mezcla se iluminó durante siete minutos con lámparas de luz fluorescente de 20 watts, posteriormente se determinó la absorbancia a $560 \mathrm{~nm}$. La actividad enzimática se reportó como U/g de PF. Una unidad de SOD es igual a la cantidad de sobrenadante que fotoinhibe el 50\% de la formación de Nitro Blue Tetrazolium Formazan (Giannopolitis y Ries 1977). 
El incremento en absorbancia debido a la formación de Nitro Blue Tetrazolium Formazan es la velocidad de reacción, la absorbancia en ausencia de SOD se usa para determinar el número de unidades $/ \mathrm{ml}$ de SOD en la solución (Stauffer 1989). Los ensayos se realizaron a temperatura ambiente.

\section{Catalasa $(C A T)$}

Para determinar la catalasa se extrajo del polvo de acetona $0,1 \mathrm{~g}$, el cual se mezcló con un homogeneizador de tejidos con $5 \mathrm{ml}$ de Tris- $\mathrm{HCl}$ frío, $0,1 \mathrm{mM}$ (pH 8,5) conteniendo $1 \%$ de polivinilpirrolidona. La mezcla se centrifugó a 12000 x g por $30 \mathrm{~min} \mathrm{a} 4^{\circ} \mathrm{C}$. La actividad de la catalasa se evaluó mediante el método descrito por Luck, citado por Blackwell et al. (1990) en el que $3 \mathrm{ml}$ de $10 \mathrm{mM}$ Tris- $\mathrm{HCl}(\mathrm{pH} 8,5)$ y $0,1 \mathrm{ml}$ de peróxido de hidrógeno $0,88 \%$ en $100 \mathrm{Mm}$ de Tris- $\mathrm{HCl}$ se colocaron en celdas cuarzo. La reacción se inició adicionando $0,1 \mathrm{ml}$ de extracto crudo y se observó el cambio en absorbancia a $240 \mathrm{~nm}$ y la actividad enzimática se reportó como $\mathrm{U} / \mathrm{g}$ de $\mathrm{PF}$, donde $\mathrm{U}=$ unidad de actividad enzimática y una unidad es igual a la descomposición de $1 \mu \mathrm{mol} / \mathrm{min}$ de $\mathrm{H}_{2} \mathrm{O}_{2}$. Los ensayos se realizaron a temperatura ambiente.

\section{Capacidad antioxidante}

La determinación del potencial antioxidante, se realizó de acuerdo con el método ABTS (2,2’azinobis (3-etilbenzotiazolin-6-ácido sulfónico) con la metodología propuesta por Rice-Evans et al. (1997) modificada por Ozgen et al. (2006), para lo cual el radical $\mathrm{ABTS}^{\bullet+}$ se formó tras la reacción de ABTS (7 $\mathrm{mM})$ con persulfato potásico $(2,45 \mathrm{mM}$, concentración final) incubados a temperatura ambiente y en oscuridad durante $16 \mathrm{~h}$. Una vez formado el radical ABTS ${ }^{++}$ este se diluyó con PBS (solución buffer de fosfato) $(\mathrm{pH} 7,4)$ hasta obtener un valor de absorbancia de 0,7 $\pm 0,1$ a $734 \mathrm{~nm}$ (longitud de máxima absorción). Para el ensayo, se mezclaron $3 \mathrm{ml}$ de la solución $\mathrm{ABTS}^{\cdot+} \mathrm{y}$ $20 \mu 1$ de extracto de la muestra, dejándose reposar por $2 \mathrm{~h}$, se realizó la lectura de absorbancia a $734 \mathrm{~nm}$. Los resultados son expresados en TEAC (actividad antioxidante equivalente a Trolox).

\section{Fenoles totales}

La cuantificación de los fenoles totales se llevó a cabo mediante el método de Folin y Ciocalteu descrito por Waterman y Mole (1994), con las siguientes modificaciones: se tomaron $0,2 \mathrm{ml}$ de solución del filtrado obtenido al realizar el polvo de acetona, al que se le adicionaron $7,8 \mathrm{ml}$ de agua desionizada y $0,5 \mathrm{ml}$ de reactivo de Folin y Ciocalteu, respectivamente, y se agitó; finalmente se agregaron 1,5 ml de solución de carbonato de sodio $20 \%$ (tiempo cero) dejándose reposar por $2 \mathrm{~h}$ en la oscuridad, y se tomó la lectura a $760 \mathrm{~nm}$ en el espectrofotómetro. La cuantificación se realizó mediante una curva patrón de ácido tánico y los resultados se reportan como $\mathrm{mg} / \mathrm{kg}$ de $\mathrm{PF}$.

\section{Vitamina C}

El cálculo del contenido de vitamina $\mathrm{C}$ se realizó de acuerdo al método de Tillman (AOAC 1990) conocido como DFI-2, 6 diclorofenol-indofenol; por el cual, se maceró una muestra de pulpa con un agente estabilizante como ácido oxálico (para mantener propia la acidez para la reacción y evitar auto-oxidación del ácido ascórbico a alto pH) y reducción del 2,6 diclorofenol-indofenol (solución de Tillman). El ácido ascórbico se estimó a partir de $5 \mathrm{~g}$ de muestra finamente picada y homogenizada con $50 \mathrm{ml}$ de ácido oxálico, tomando una alícuota de $10 \mathrm{ml}$.

\section{Análisis estadístico}

Se realizó un análisis comparación de medias mediante la prueba de Tukey $(p=0,05)$, en la que se empleó el paquete de análisis estadístico SAS (2002).

\section{RESULTADOS Y DISCUSIÓN}

\section{Peroxidasa (POD)}

Se encontró que a los tres días de almacenamiento (dda) (Cuadro 1), la actividad de la enzima peroxidasa (POD) en los tratamientos a $10^{\circ} \mathrm{C}$ y testigo superaron 
Cuadro 1. Actividad enzimática de peroxidasa, polifenol oxidasa, superóxido dismutasa y catalasa en menta almacenada bajo refrigeración $\left(6\right.$ y $\left.10^{\circ} \mathrm{C}\right)$ y en condiciones de temperatura ambiente. Texcoco, Estado de México, México. 2011.

\begin{tabular}{|c|c|c|c|c|}
\hline \multirow{2}{*}{$\begin{array}{l}\text { Temperatura de almacenamiento } \\
\qquad\left({ }^{\circ} \mathrm{C}\right)\end{array}$} & POD & PFO & SOD & CAT \\
\hline & (U/g de PF) & (U/g de PF ) & (U/g de PF ) & ( U/g de PF ) \\
\hline & \multicolumn{4}{|c|}{ o días de almacenamiento } \\
\hline & $194,72 *$ & 58,72 & 53,68 & 23,16 \\
\hline & \multicolumn{4}{|c|}{3 días de almacenamiento } \\
\hline 6 & $111,01 \mathrm{~b}$ & $95,15 \mathrm{~b}$ & 33,52 a & $23,09 \mathrm{a}$ \\
\hline 10 & $621,20 \mathrm{a}$ & $94,59 \mathrm{~b}$ & 33,34 a & 44,07 a \\
\hline Ambiente & 387,60 a & $116,42 \mathrm{a}$ & 34,39 a & 42,38 a \\
\hline \multirow[t]{2}{*}{ DMSH } & 234,86 & 14,09 & 7,91 & 34,75 \\
\hline & \multicolumn{4}{|c|}{6 días de almacenamiento } \\
\hline 6 & $234,30 \mathrm{a}$ & $111,83 \mathrm{a}$ & $38,66 \mathrm{a}$ & $25,75 \mathrm{a}$ \\
\hline 10 & $174,96 \mathrm{ab}$ & $98,06 \mathrm{~b}$ & $36,30 \mathrm{a}$ & $21,71 \mathrm{a}$ \\
\hline Ambiente & $141,72 \mathrm{~b}$ & $106,30 \mathrm{a}$ & $31,24 \mathrm{a}$ & 18,19 a \\
\hline \multirow[t]{2}{*}{ DMSH } & 89,68 & 6,09 & 9,79 & 18,10 \\
\hline & \multicolumn{4}{|c|}{9 días de almacenamiento } \\
\hline 6 & $166,32 \mathrm{a}$ & $108,21 \mathrm{a}$ & $27,46 \mathrm{ab}$ & $11,28 \mathrm{a}$ \\
\hline 10 & $98,78 \mathrm{a}$ & $98,26 \mathrm{a}$ & 30,47 a & $14,72 \mathrm{a}$ \\
\hline Ambiente & 138,18 a & 121,59 a & $24,28 \mathrm{~b}$ & $11,35 \mathrm{a}$ \\
\hline \multirow[t]{2}{*}{ DMSH } & 97,13 & 24,29 & 5,22 & 15,65 \\
\hline & \multicolumn{4}{|c|}{12 días de almacenamiento } \\
\hline 6 & $231,48 \mathrm{a}$ & $114,29 \mathrm{~b}$ & 40,97 a & 20,91 a \\
\hline 10 & $208,60 \mathrm{a}$ & $101,80 \mathrm{c}$ & 35,37 a & 29,15 a \\
\hline Ambiente & $292,56 \mathrm{a}$ & $190,68 \mathrm{a}$ & $25,32 \mathrm{~b}$ & $24,24 \mathrm{a}$ \\
\hline \multirow[t]{2}{*}{ DMSH } & 102,95 & 11,77 & 6,61 & 14,86 \\
\hline & \multicolumn{4}{|c|}{15 días de almacenamiento } \\
\hline 6 & $66,73 \mathrm{c}$ & $90,02 \mathrm{a}$ & $26,22 \mathrm{a}$ & $15,00 \mathrm{a}$ \\
\hline 10 & 306,29 a & $86,90 \mathrm{a}$ & $20,69 a b$ & $13,57 \mathrm{a}$ \\
\hline Ambiente & $162,50 \mathrm{~b}$ & $84,33 \mathrm{a}$ & $17,89 \mathrm{~b}$ & $11,56 \mathrm{a}$ \\
\hline DMSH & 43,39 & 15,15 & 7,19 & 15,65 \\
\hline
\end{tabular}

POD: peroxidasa (U/g de PF); PFO: polifenol oxidasa (U/g de PF); SOD: superóxido dismutasa (U/g de PF); CAT: catalasa (U/g de PF). * Aquí aun no hay efecto de tratamientos, solo se reporta un valor medio como referencia.

Medias con la misma letra dentro de columnas, son iguales de acuerdo con la prueba de Tukey con $\mathrm{P}=0,05$.

DMSH: Diferencia mínima significativa honesta.

estadísticamente $(\alpha=0,05)$ al de $6^{\circ} \mathrm{C}$, cuyos valores máximos fueron 621,20 y $387,60 \mathrm{U} / \mathrm{g}$ de $\mathrm{PF}$, respectivamente. Estos incrementos pueden estar asociados a la generación de un estrés oxidativo provocado por la exposición del área foliar a condiciones no favorables de temperatura, por lo que se puede generar una mayor 
liberación de la POD al citoplasma, incrementando su actividad. Este comportamiento en POD ha sido mencionado por Goodman et al. (1986) y recientemente confirmado por Altunkaya y Gökmen (2008) y Ding et al. (2009). Por otra parte, se ha reportado que bajo condiciones de almacenamiento en frío la actividad de la POD disminuye (Thompson 2003), lo cual coincidió con lo observado en la menta almacenada a $6^{\circ} \mathrm{C}$.

A los 6 dda en frío $\left(6\right.$ y $\left.10^{\circ} \mathrm{C}\right)$ la actividad de la POD en la menta fue significativamente superior a lo observado a temperatura ambiente con valores de 234,30 y 174,96 U/g de PF, respectivamente. Estos resultados sugieren que la POD es una molécula clave en la aclimatación de la menta o de alguno de sus órganos a los cambios que experimenta el medio ambiente en el que se desarrolla.

También se detectaron efectos significativos $(\alpha=0,05)$ en el último periodo de muestreo, donde todos los tratamientos de almacenamiento evaluados fueron diferentes entre sí, destacando el tratamiento a $10^{\circ} \mathrm{C}$ sobre los de $6^{\circ} \mathrm{C}$ y temperatura ambiente, con actividades de $306,29,162,50$ y $66,73 \mathrm{U} / \mathrm{g}$ de PF, respectivamente. No se ha logrado determinar en forma clara el papel de la POD en el proceso de senescencia, ya que se ha observado que en hojas de hierbas como tomillo aguja (Thymbra spicata L. var. spicata) y algunas especies de salvia, la actividad de esta enzima disminuye al aumentar la temperatura (Dogan et al. 2007a, 2007b); mientras que en frutos como papaya, su comportamiento fue inverso (Sankat y Maharj 1997). La disminución que se observó en la actividad de la POD en el tratamiento testigo $\left(20 \pm 1^{\circ} \mathrm{C}\right)$, contrasta con Alia et. al. (2002), quienes encontraron que en frutos de zapote mamey (Pouteria sapota Jacq. H.E. Moore \& Stearn) almacenados a $15^{\circ} \mathrm{C}$ se incrementó de manera considerable la actividad de esta enzima.

\section{Polifenol oxidasa (PFO)}

Se observó que a los tres días de almacenamiento (Cuadro 1), el tratamiento testigo, mostró mayor actividad de la polifenol oxidasa (PFO) con 116,42 U/g de $\mathrm{PF}$ en comparación a los tratamientos a 6 y $10^{\circ} \mathrm{C}$, los cuales presentaron actividades estadísticamente similares de 95,15 y 94,59 U/g de PF, respectivamente; estos resultados indican que la temperatura del medio ambiente afectó negativamente a los tejidos, provocando el inicio de su deterioro. $\mathrm{Al}$ respecto, Trujillo et al. (2006) y Hassanpour et al. (2012), mencionan que esta enzima se encuentra localizada exclusivamente en plastidios y es liberada al citososol como consecuencia de un daño o senescencia.

Al sexto día de almacenamiento se encontró un efecto significativo $(\alpha=0,05)$ de la temperatura sobre la actividad de la PFO en los tratamientos $6^{\circ} \mathrm{C}$ y temperatura ambiente, en relación al de $10^{\circ} \mathrm{C}$. Este efecto es contrario a lo reportado por Kravayan y Aydemir (2001), quienes al purificar y caracterizar a esta enzima en hojas de menta (Mentha piperita L.) indican que la actividad de la PFO es estable a bajas temperaturas; estos mismos autores mencionan que existe una correlación positiva con el incremento de la temperatura. Por lo que se sugiere que el comportamiento de estos resultados podrían encontrarse estrechamente vinculados con la presencia de isoenzimas, las cuales pueden estar actuando bajo condiciones diferentes de temperatura (Concellón et al. 2004, Neves et al. 2009, Hassanpour et al. 2012).

Durante el penúltimo periodo de muestreo, se observó que la actividad de la PFO en el tratamiento testigo aumentó considerablemente, puesto que su valor máximo fue de 190,68 U/g de PF, en relación a lo que se presentó a 6 y $10^{\circ} \mathrm{C}$ con valores de 114,29 y 101,80 U/g de PF, respectivamente; esto concuerda con lo indicado por varios autores en hierbas aromáticas como el orégano (Origanum vulgare ssp. Hirtum) (Dogan et al. 2005), menta poleo (Mentha pulegium) (Hassanpour et al. 2012), tomillo (Thymus longicaulis subsp. Chaubardii var. chaubardii) (Dogan y Dogan 2004) y algunos frutos como pitahaya amarilla (Acanthocereus pitahaja) (Baquero et al. 2005) y uva (Vitis vinífera ssp. Sativa) (Rapeanu et al. 2006).

\section{Superóxido dismutasa (SOD)}

Durante los dos primeros muestreos (tres y seis días), la actividad enzimática de la superóxido dismutasa (SOD) no presentó cambios significativos $(\alpha=0,05)$ entre tratamientos, sino hasta al día 9 de almacenamiento, en donde los tratamientos a bajas temperaturas $\left(6\right.$ y $\left.10^{\circ} \mathrm{C}\right)$ mostraron valores estadísticamente más altos de 27,46 y 30,47 U/g de PF comparados con el de ambiente, que fue de $24,28 \mathrm{U} / \mathrm{g}$ de PF (Cuadro 1). Con respecto a estos resultados, se ha encontrado que la resistencia de las plantas a bajas temperaturas se encuentra asociada 
a la actividad conjunta de las enzimas superóxido dismutasa y catalasa, más que a otros factores fisiológicos (Scandalios 1993, Polata et al. 2009), ya que constituyen la primera línea de defensa celular en contra de las especies reactivas de oxígeno (ERO) (del Rio et al. 2003, Ferreira-Silva et al. 2012). Por lo que se sugiere que un incremento en su actividad enzimática puede resultar benéfica (Alsher et al. 2002, Palma et al. 2006, Vranová et al. 2002).

Se encontró un incremento en la actividad de la SOD a los doce días de almacenamiento, en la que los tratamientos con frío $\left(6\right.$ y $\left.10^{\circ} \mathrm{C}\right)$ fueron estadísticamente superiores a los de temperatura ambiente, con actividades de 40,97 y 35,37 U/g de PF, respectivamente, lo cual concuerda con Cantwell y Reid (2002) y Mi et al. (2008) quienes indican que algunas hierbas aromáticas para mercado fresco como perejil, berro y menta no son susceptibles a daño por frío por lo que comúnmente se enfrían con hielo. Por consiguiente, los resultados encontrados podrían suponer que un incremento en la actividad de la SOD durante el almacenamiento a 6 y $10^{\circ} \mathrm{C}$ puede conferir una protección contra un posible daño a la integridad de la membrana celular, lo cual coincide con Cuadra-Crespo y del Amor (2010) quienes al evaluar la actividad enzimática de la SOD en frutos de chile pimiento morrón almacenados bajo condiciones de frío y de alta humedad relativa, encontraron un incremento de su actividad sin que se hubiese observado algún tipo de daño en su apariencia.

En el último periodo de muestreo el comportamiento de la SOD fue similar al del día 9 de almacenamiento, donde de igual manera los tratamientos a bajas temperaturas $\left(6 \mathrm{y} 10^{\circ} \mathrm{C}\right)$ fueron estadísticamente superiores $(\alpha=0,05)$ a lo encontrado con la temperatura de almacenamiento testigo, con valores de 26,22 y 20,69 $\mathrm{U} / \mathrm{g}$ de PF, respectivamente. Con respecto a lo anterior, se podría inducir que bajo esas temperaturas de almacenamiento, al menos una de las enzimas (SOD) que apoyan la disminución de la senescencia es activada por el estrés oxidativo en las hojas de menta. Este mismo comportamiento ha sido mencionado en menta poleo (Mentha pulegium) (Candan y Tarthan 2003, Oueslati et al.2010) y en frutos como pitahaya (Hylocereus undatus) (Balois et al. 2008) y pitaya amarilla (Acanthocereus pitajaya) (Baquero et al. 2005).

\section{Catalasa (CAT)}

La actividad de catalasa (CAT) no presentó diferencias significativas $(\alpha=0,05)$ entre tratamientos durante el periodo de almacenamiento (Cuadro 1), lo que puede sugerir que esta enzima es estable a las temperaturas empleadas $\left(6,10^{\circ} \mathrm{C}\right.$ y ambiente), y coincide con Balois et al. (2008) quienes reportan para frutos de pitaya, que la actividad de CAT es insensible al frío y a condiciones de manejo a temperatura ambiente. En este mismo sentido, Sala y Lafuente (2000) indican que en frutos como la mandarina, la CAT es una enzima antioxidante involucrada en los mecanismos de protección contra el estrés por frío. En contraste a lo observado, en hojas maduras de nuez vómica (Strychnos nux-vomica L.), Vijayakumar et al. (2009), quienes determinaron una alta actividad de la CAT. Lo anterior indica que estas diferencias pueden ser dependientes de la especie, momento fisiológico de cosecha, tiempo de almacenamiento, entre otros factores.

\section{Capacidad antioxidante}

Se encontró que al tercer día de almacenamiento, el tratamiento a $10^{\circ} \mathrm{C}$ mostró el mayor valor de capacidad antioxidante $(403,36 \mathrm{~mm}$ TEAC/g de PF) con relación a $6^{\circ} \mathrm{C}$ y testigo, los cuales fueron estadísticamente similares con 283,41 y $332,45 \mathrm{~mm}$ TEAC/g de $\mathrm{PF}$, respectivamente (Cuadro 2). $\mathrm{Al}$ respecto se ha indicado que la actividad antioxidante de las hierbas aromáticas se debe a la presencia de grupos hidroxilo en los compuestos fenólicos, donde las condiciones de almacenamiento a bajas temperaturas pueden favorecer su expresión, como lo señalan Ayala-Zavala et al. (2004) y Jin et al. (2011), para fresa manejada a $10^{\circ} \mathrm{C}$ en la que se incrementó la capacidad antioxidante y la producción de compuestos aromáticos.

También se presentaron diferencias estadísticas al día 15 de almacenamiento, donde los tratamientos a $10^{\circ} \mathrm{C}$ y a temperatura ambiente mostraron los valores más altos de capacidad antioxidante con 327,32 y $291,87 \mathrm{~mm} \mathrm{TEAC} / \mathrm{g}$ de PF comparado a lo que se observó a $6^{\circ} \mathrm{C}$ con $261,11 \mathrm{~mm}$ TEAC/g de PF. Al comparar estos resultados con los mencionados por Gil et al. (2011) para Mentha piperita L., fueron similares, 
Cuadro 2. Capacidad antioxidante, contenido de fenoles totales y vitamina $\mathrm{C}$ en menta almacenada bajo refrigeración $\left(6 \mathrm{y} 10^{\circ} \mathrm{C}\right)$ y en condiciones de temperatura ambiente. Texcoco, Edo de México, México. 2011.

\begin{tabular}{|c|c|c|c|}
\hline $\begin{array}{c}\text { Temperatura de } \\
\text { almacenamiento }\left({ }^{\circ} \mathrm{C}\right)\end{array}$ & $\begin{array}{c}\text { Capacidad antioxidante } \\
(\mathbf{m m} \text { TEAC/g de PF) }\end{array}$ & $\begin{array}{c}\text { Fenoles totales } \\
(\mathrm{mg} / \mathrm{kg} \text { de } \mathbf{P F})\end{array}$ & $\begin{array}{c}\text { Vitamina C } \\
(\mathrm{mg} \text { ac, ascórbico/100 g) }\end{array}$ \\
\hline & \multicolumn{3}{|c|}{0 días de almacenamiento } \\
\hline & $262,57 *$ & 12,19 & 22,17 \\
\hline & \multicolumn{3}{|c|}{3 días de almacenamiento } \\
\hline 6 & $283,41 \mathrm{~b}$ & $12,93 \mathrm{ab}$ & $36,73 \mathrm{a}$ \\
\hline 10 & 403,36 a & $19,62 \mathrm{a}$ & $33,42 \mathrm{a}$ \\
\hline Ambiente & $332,45 \mathrm{~b}$ & $9,90 \mathrm{~b}$ & $35,03 \mathrm{a}$ \\
\hline \multirow[t]{2}{*}{ DMSH } & 49,59 & 8,33 & 5,94 \\
\hline & \multicolumn{3}{|c|}{6 días de almacenamiento } \\
\hline 6 & 229,48 a & $8,86 \mathrm{~b}$ & 36,17 a \\
\hline 10 & 204,85 a & $9,10 \mathrm{~b}$ & 38,37 a \\
\hline Ambiente & 219,09 a & 14,03 a & 40,17 a \\
\hline \multirow[t]{2}{*}{ DMSH } & 50,01 & 6,09 & 11,35 \\
\hline & \multicolumn{3}{|c|}{9 días de almacenamiento } \\
\hline 6 & 308,47 a & $14,35 \mathrm{a}$ & 31,17 a \\
\hline 10 & 355,06 a & 12,66 a & 32,17 a \\
\hline Ambiente & 305,19 a & $8,28 \mathrm{a}$ & $30,42 \mathrm{a}$ \\
\hline \multirow[t]{2}{*}{ DMSH } & 51,75 & 6,09 & 10,25 \\
\hline & \multicolumn{3}{|c|}{12 días de almacenamiento } \\
\hline 6 & 307,90 a & 18,31 a & 35,92 a \\
\hline 10 & 334,37 a & $16,06 \mathrm{ab}$ & $30,41 \mathrm{a}$ \\
\hline Ambiente & 300,75 a & $11,89 \mathrm{~b}$ & 30,91 a \\
\hline \multirow[t]{2}{*}{ DMSH } & 74,15 & 6,32 & 6,39 \\
\hline & \multicolumn{3}{|c|}{15 días de almacenamiento } \\
\hline 6 & $261,11 \mathrm{~b}$ & 18,81 a & 39,67 a \\
\hline 10 & $327,32 \mathrm{a}$ & 17,77 a & $30,17 \mathrm{~b}$ \\
\hline Ambiente & $291,87 \mathrm{ab}$ & $9,52 \mathrm{~b}$ & $34,42 a b$ \\
\hline DMSH & 35,94 & 6,63 & 8,82 \\
\hline
\end{tabular}

* Aquí aun no hay efecto de tratamientos, solo se reporta media como referencia.

Medias con la misma letra dentro de columnas, son iguales de acuerdo con la prueba de Tukey con P=0,05. DMSH: diferencia mínima significativa honesta.

con valores de $332,3 \mathrm{~mm}$ TEAC/g de PF, mientras que fueron mayores a lo señalado para hierbabuena (Mentha spicata L.) (Rodríguez et al. 2006, Tawaha et al. 2007). Sin embargo, resulta difícil comparar resultados relacionados con la capacidad antioxidante, debido a que se reportan bajo diferentes métodos de estimación, así como por el estado y manejo general de la muestra (Rice-Evans et al. 1997, Re et al. 1999, 
Campanella et al. 2003, Vargas et al. 2005, Scalzo et al. 2005, Kirca y Arslan 2008).

\section{Fenoles totales}

En lo que se refiere al contenido de fenoles totales (FT) (Cuadro 2), se puede observar que en el día 3 de almacenamiento, los tratamientos a 6 y $10^{\circ} \mathrm{C}$ mostraron valores estadísticamente $(\alpha=0,05)$ similares de 12,93 y $19,62 \mathrm{mg} / \mathrm{kg}$ de PF; sin embargo, este último fue superior al testigo $(9,90 \mathrm{mg} / \mathrm{kg}$ de $\mathrm{PF})$, comportamiento que se puede asociar con un incremento de la actividad antioxidante, debido a que los fenoles en conjunto con las enzimas superóxido dismutasa, catalasa, entre otras, constituyen la primera línea de defensa contra el estrés oxidativo inducido por las bajas temperaturas, manteniendo estable la integridad de la membrana celular (Kaur y Kapoor 2001, Dorman et al. 2003, Kevers et al. 2007, Restrepo et al. 2009, Kirca y Arslan 2008). Sin embargo, también se ha reportado que la exposición de frutas y hortalizas en condiciones de almacenamiento entre 0 y $15^{\circ} \mathrm{C}$ puede provocar un oscurecimiento en sus tejidos por la acción de la enzimas polifenol oxidasa y peroxidasa sobre los fenoles (Balois et al. 2008); no obstante, se ha mencionado que la menta es un cultivo insensible a daños por frío (Cantwell y Reid 1993, Cantwell y Reid 2002).

Por otra parte, se encontró que a los seis días de almacenamiento (dda) el contenido de FT, tuvo un comportamiento contrario a los demás días de evaluación, ya que el tratamiento testigo superó a los de 6 y $10^{\circ} \mathrm{C}$. En relación con lo anterior, Wojdylo et al. (2007) y Khalaf et al. (2008), indican que la estabilidad de los compuestos fenólicos está en función de la temperatura de almacenamiento, luz y la disposición de oxígeno; y que en condiciones de temperatura ambiental, estos tienden a mostrar un comportamiento poco uniforme entre las especies de plantas analizadas, aún entre genotipos de la misma especie (PiljacŽegarac y Šamec 2011).

A los $12 \mathrm{dda}$, se observó que el tratamiento a $6^{\circ} \mathrm{C}$ fue estadísticamente $(\alpha=0,05)$ superior al testigo con un valor de $18,31 \mathrm{mg} / \mathrm{kg}$ de $\mathrm{PF}$, lo cual es similar a resultados reportados por Cantwell et al. (2002) quienes en raíces de jícama almacenada en condiciones de frío, indican un incremento en el contenido de fenoles.

Al final del periodo de evaluación (15 dda), se pudo apreciar que los tratamientos a bajas temperaturas de almacenamiento $\left(6\right.$ y $\left.10^{\circ} \mathrm{C}\right)$ presentaron el mayor contenido de FT $(18,81$ y $17,77 \mathrm{mg} / \mathrm{kg}$ de PF, respectivamente), en relación con lo que se presentó el testigo, siendo este resultado inverso a lo mostrado a los $6 \mathrm{dda}$, por lo que se alude un mayor efecto benéfico del frío en la disminución de los procesos fisiológicos que conducen a la senescencia (Do Nascimento y Pierre 2003), lo cual nuevamente confirman lo reportado por Cantwell y Reid (1993 y 2002). Sin embargo, no se han encontrado investigaciones en menta que indiquen un incremento en la concentración de fenoles totales por efecto del daño por frío o a largos periodos de almacenamiento, como se señala para frutos como zapote mamey (Pouteria sapota (Jacq.) H.E. Moore and Stearn) (Alia et al. 2005), mango (Mangifera indica) y guayaba (Psidium gujava) (Patthamakanokporn et al. 2008).

\section{Vitamina C}

De acuerdo con la comparación de medias (Cuadro 2), el contenido de vitamina $\mathrm{C}$ (ácido ascórbico) no presentó diferencias significativas $(\alpha=0,05)$ durante la mayor parte del periodo de almacenamiento, a excepción del último muestreo, donde el tratamiento a $6^{\circ} \mathrm{C}$ fue significativamente superior al testigo. En relación con esta fecha de evaluación se ha mencionado que el almacenamiento a bajas temperaturas se considera eficiente en la reducción de las pérdidas de ácido ascórbico, incluso la congelación suele ser aún mejor (Rapisarda et al. 2008, Tavarini et al. 2008), y al ser este un compuesto no enzimático que contribuye a la capacidad antioxidante, su conservación es importante durante el manejo poscosecha de frutas y hortalizas (Velioglu et al. 1998, Shivashankara et al. 2004).

La disminución en el contenido de vitamina $\mathrm{C}$ en la menta almacenada a temperatura ambiente puede ser asociada con un incremento en la tasa de respiración, debido a que la tasa de cambios bioquímicos causados por procesos fisiológicos, entre ellos el respiratorio, se incrementan con las altas temperaturas $(\mathrm{Ca}-$ net y Álvarez 2006), y al ser este un proceso del tipo oxidativo genera especies reactivas de oxígeno, por lo que enzimas como la ascorbato peroxidasa y ascorbato reductasa encargadas de detoxificar a la célula, pueden jugar un papel importante al usar el ácido ascórbico como sustrato, con la consecuente disminución de sus niveles (Camarena 2006, Viña y Chaves 2006). 


\section{LITERATURA CITADA}

Alia, TI; Colinas, LMT; Martínez, DMT; Soto, HMR. 2002. Factores fisiológicos, bioquímicos y de calidad en frutos de zapote mamey (Pouteria sapota Jacq. H.E. Moore \& Stearn) durante poscosecha. Revista Chapingo Serie Horticultura 8:263-281.

Alia, TI; Colinas, LMT; Martínez, DMT; Soto, HMR. 2005. Daños por frío en zapote mamey (Pouteria sapota (Jacq.) H.E. Moore \& Stearn) II: Cambios en fenoles totales y actividad enzimática. Revista Fitotecnia Mexicana 28:25-32.

Alsher, GR; Erturk, N; Heath, SL. 2002. Role of superoxide dismutases (SODs) in controlling oxidative stress in plants. Journal Experimental Botany 53:1331-1341.

Altunkaya, A; Gökmen, V. 2008. Effect of various inhibitors on enzymatic browning, antioxidant activity and total phenol content of fresh lettuce (Lactuca sativa). Food Chemistry 107:1173-1179.

Anónimo. 1988. Enciclopedia de los municipios de México. Vol. 5. Municipios de Morelos. Secretaria de Gobernación. Instituto Nacional para el Federalismo y el Desarrollo Municipal. D.F., México. 649 p.

AOAC (Association of Official Analytical Chemistry). 1990. Official methods of analysis of the Association Analytical Chemistry. 14 ed. Published for the association of Official Analytical Chemists Inc. Arlington, VA. USA. 1006 p.

Ayala-Zavala, JF; Wang, SY; Wang, CY; González-Aguilar, GA. 2004. Effect of storage temperatures on antioxidant capacity and aroma compounds in strawberry fruit. Food Science and Technology 37:687-695.

Balois, MR; Colinas, LMT; Peña, VCB; Chávez, FSH; Alia, TI. 2008. Sistema enzimático antisenescencia catalasa-superóxido dismutasa, de frutas de pitahaya (Hylocereus undatus) almacenados en frío. Revista Chapingo Serie Horticultura 14:295-299.

Baquero, DLE; Castro, RJA; Narváez, CCE 2005. Catalasa, peroxidasa y polifenoloxidasa en pitaya amarilla (Acanthocereus pitajaya): maduración y senescencia. Acta Biológica Colombiana 10:49-59.

Beyer, FW; Fridovich, I. 1987. Assaying for superoxide dismutase activity: some large consequences of minor changes in conditions. Analytical Biochemistry 161:559-566.

Blackwell, RD; Murray, AJS; Lea, PJ. 1990. Enzymes of photorespiratory carbon pathway. In Lea, PJ. ed. Methods in plant biochemistry. Academic Press. USA. p. 129-144.
Camarena, GG. 2006. Las especies reactivas de oxígeno en defensa de las plantas contra patógenos. Revista Chapingo: Serie Ciencias Forestales y del Ambiente 12:25-30.

Campanella, L; Bonani, A; Favero, G; Tomasseti, M. 2003. Determination of antioxidant properties of aromatic herbs, olives and fresh fruit using an enzymatic sensor. Analytical and Bioanalytical Chemistry 375:1011-1016.

Candan, N; Tarthan, L. 2003. The correlation between antioxidant enzyme activities and lipid peroxidation levels in Mentha pulegium organs grown in $\mathrm{Ca}^{2+}$, $\mathrm{Mg}^{2+}, \mathrm{Zn}^{2+}$ and $\mathrm{Mn}^{2+}$ stress conditions. Plant Science 165:769-776.

Canet, PW; Álvarez, TMD. 2006. Quality and safety of frozen vegetables. In Sun, WD. ed. Handbook of frozen food processing and packaging. Taylor \& Francis Group. LLC. Florida, USA. p. 377-410.

Cantwell, MI; Reid, MS. 1993. Postharvest physiology and handling of fresh culinary herbs. Journal Herbs Spices \& Medicinal Plants 1:93-127.

Cantwell, MI; Peiser, G; Mercado-Silva, E. 2002. Induction of chilling injury in jicama (Pachyrhizus erosus) roots: changes in texture, color and phenolics. Postharvest Biology and Technology 25:311-320.

Cantwell, MI; Reid, MS. 2002. Postharvest handling systems: fresh herbs. In Kader, A. ed. Postharvest technology of horticultural crops. Chapter 26. University of California. California, USA. p. 367-372.

Concellón, M; Añon, Mc; Chaves, AR. 2004. Characterization and changes in polyphenol oxidase from eggplant fruit (Solanum melogena L.) during storage at low temperature. Food Chemistry 88:17-24.

Cuadra-Crespo, P; del Amor, MF. 2010. Effects of postharvest treatments on fruit quality of sweet pepper at low temperature. Journal of the Science of Food and Agriculture 90:2716-2722.

del Rio, AL; Sandalio, ML; Altomare, AD; Zilinskas, AB. 2003. Mitocondrial and peroxisomal manganese superoxide dismutase: differential expression during leaf senescence. Journal of Experimental Botany 54:923-933.

Ding, Z; Tian, S; Meng, X; Xu, Y. 2009. Hydrogen peroxide is correlated with browning in peach fruit stored at low temperature. Frontiers of Chemical Engineering in China 3:363-374.

Djeridane, A; Yousfi, M; Nadjemi, B; Boutassouna, D; Stocker, P; Vidal, N. 2006. Antioxidant activity of some Algerian medicinal plants extracts containing phenolic compounds. Food Chemistry 53:4290-4302. 
Do Nascimento, NMC; Pierre, EJ. 2003. Storage temperature. Chapter 8. In Bartz A, J; Brencht K, J. eds. Postharvest physiology and pathology of vegetables. Marcel Dekker, Inc. New York, USA. p. 1-20.

Dogan, S; Dogan, M. 2004. Determination of kinetic properties of polyphenol oxidase from from Thymus (Thymus longicaulis subsp. chaubardii var. chaubardii). Food Chemistry 88:69-77.

Dogan, S; Arslan, O; Özen, F. 2005. Polyphenoloxidase activity of oregano at different stages. Food Chemistry 91:341-345.

Dogan, S; Turan, P; Dogan, M; Arslan, O; Alkan, M. 2007a. Partial characterization of peroxidase from leaves of thymbra plant (Thymbra spicata L. var. spicata). European Food Research and Technology 225:865-871.

Dogan, S; Turan, P; Dogan, M; Arslan, O; Alkan, M. 2007b. Variations of peroxidase activity among Salvia species. Journal of Food Engineering 79:375-382.

Dorman, DHJ; Kosar, M; Kahlos, K; Holm, Y; Hiltunen, R. 2003. Antioxidant properties and composition of aqueous extracts from mentha species, hybrids, varieties and cultivars. Journal of Agricultural and Food Chemistry 51:4563-4569.

Erdemoglu, N; Turan, NN; Cakici, I; Sener, B; Aydin, A. 2006. Antioxidant activities of some Lamiaceae plant extracts. Phytotherapy Research 20:9-13.

Farmacopea Europea IV. 2002. European pharmacopoeia. 4 ed. Strasbourg: Cedex. Council of Europe. Strasbourg, France. 3263 p.

Ferreira-Silva, SL; Voigt, EL; Silva, EN; Maia, JM; Aragáo, TCR; Silveira, JAG. 2012. Partial oxidative protection by enzymatic and non-enzymatic components an cashew under high salinity. Biologia Plantarum 56:172-176.

Flurkey, WH; Jen, J. 1978. Peroxidase and polyphenoloxidase activities in developing peaches. Journal of Food Science 43:1828-1831.

Giannopolities, CN; Ries, SK. 1977. Superoxide dismutases. Plant Physiology 59:309-314.

Gil, DMA; Falé, PLV; Serralheiro, MLM; Rebelo, MJF. 2011. Herbal infusion bio electrochemical polyphenolic index: Green tea - The gallic acid interference. Food Chemistry 129:1537-1543.

Goodman, RN; Kiraly, Z; Wood, KR. 1986. Secondary metabolite. In Goodman, RN. ed. The biochemistry and physiology of plant disease. Missouri: University of Missouri. USA. p. 221-224.
Hassanpour, H; Khavari-Nejad, A; Nikman, V; Najafi, F; Razavi, K. 2012. Effects of penconazole and water deficit stress on physiological and antioxidative responses in pennyroyal (Mentha pulgium L.). Acta Physiologiae Plantarum 34:1537-1549.

Jin, P; Wang, YS; Wang, YC; Zheng, Y. 2011. Effect of cultural system and storage temperature on antioxidant capacity and phenolic compounds in strawberries. Food Chemistry 124:262-270.

Karousou, R; Balta, M; Hanlidou, E; Kokkini, S. 2007. "Mints", smells and traditional uses in Thessaloniki (Greece) and others Mediterranean countries. Journal of Ethnopharnacology 109:248-257.

Kaur, C; Kapoor, HC. 2001. Antioxidants in fruits and vegetables: the millennium's health. International Journal of Food Science and Technology 36:703-725.

Kavrayan, D; Aydemir, T. 2001. Partial purification and characterization of polyphenoloxidase from peppermint (Mentha piperita). Food Chemistry 74:147-154.

Kevers, C; FalkowskI, M; Tabart, J; Defraigne, JO; Dommes, J; Pincemail, J. 2007. Evolution of antioxidant capacity during storage of selected fruits and vegetables. Journal of Agricultural and Food Chemistry 55:8596-8603.

Khalaf, AN; Skakya, AK; Al-Othman, A; El-Agbar, Z; Farah, H. 2008. Antioxidant activity of some common plants. Turkish Journal of Biology 32:51-55.

Kirca, A; Arslan, E. 2008. Antioxidant capacity and total phenolic content of selected plants from Turkey. International Journal of Food Science and Technology 43:2038-2046.

Laminkanra, O. 1995. Enzymatic browning of muscadine grapes products. In Lee, CL; Whitaker, JR. eds. Enzymatic browning and its prevention. ACS. Washington DC., USA. p. 166-177.

Mi, YK; Hwan, LC; Lee, H; Moon, B; Yong, LC. 2008. Relative antioxidant and cytoprotective activities of common herbs. Food Chemistry 106:929-939.

Miliauskas G; Venskutonis, PR; van Beek TA. 2004. Screening of radical scavenging activity of some medicinal and aromatic plant extracts. Food Chemistry 85:231-237.

Mimica-Dukin, N; Bozin, B. 2008. Mentha L. species (Lamiaceae) as promising sources of bioactive secondary metabolites. Current Pharmaceutical Design 14:31413150 .

Neves, AV; Pichii, GD; Da Silva, AM. 2009. Some biochemical properties of polyphenoloxidase from spearmint 
(Mentha arvensis). Brazilian Archives of Biology and Technology 52:1001-1010.

Nickavar, B; Alinaghi, A; Kamalinejad, M. 2008. Evaluation of the antioxidant properties of five mentha species. Iranian Journal of Pharmaceutical Research 7:203209.

Oueslati, S; Karray-Bouraoui, N; Attia, H; Rabhi, M; Ksouri, R; Lachal, M. 2010. Physiological and antioxidant responses of Mentha pulgium (Pennyroyal) to salt stress. Acta Physiologiae Plantarum 32:289-296.

Ozgen, M; Reese, NR; Tulio Jr, ZA; Scheerens, CJ; Miller, RA. 2006. Modified 2,2-Azino-bis-3-ethylbenzothiazoline-6-sulfonic acid (ABTS) method to measure antioxidant capacity of selected small fruits and comparison to ferric reducing antioxidant power (FRAP) and 2,2'-diphenil-1-picrylhydrazyl (DPPH) methods. Journal of Agricultural and Food Chemistry 54:1151-1157.

Palma, MJ; Jiménez, A; Sandalio, ML; Corpas, JF; Lundqvist, M; Gómez, M; Sevilla, F; del Rio, AL. 2006. Antioxidative enzymes from chloroplast, mitochondria, and peroxisomes during senescence of nodulated pea plants. Journal of Experimental Botany 57:1747-1758.

Patthamakanokporn, O; Puwastien, P; Nitithamyong, A; Sirichakwal, PP. 2008. Changes of antioxidant activity and total phenolic compounds during storage of selected fruits. Journal of Food Composition and Analysis 21:241-248.

Piljac-Žegarac, J; Šamec, D. 2011. Antioxidant stability of small fruits in postharvest storage at room and refrigerator temperatures. Food Research International 44:345-350

Polata, H; Wiliniska, A; Bryjak, J; Polakovic, M. 2009. Thermal inactivation kinetics of vegetable peroxidases. Journal of Food Engineering 91:387-391.

Rapeanu, G; Van Loey, A; Smout, C; Hendrickx, M. 2006. Biochemical characterization and process stability of polyphenoloxidase extracted from Victoria grape ( $\mathrm{Vi}$ tis vinifera ssp. Sativa). Food Chemistry 94:253-261.

Rapisarda, P; Blanco, IM; Pannuzzo, P; Timparo, N. 2008. Effect of cold five orange genotypes (Citrus sinensis (L.) Osbeck). Postharvest Biology and Technology 49:348-354.

Restrepo, DC; Narváez, CE; Restrepo, LP. 2009. Extracción de compuestos con actividad antioxidante de frutos de guayaba cultivada en Vélez-Santander, Colombia. Química Nova 32:1517-1522.

Re, R; Pellegreni, N; Proteggente, A; Pannala, A; Yang, M; Rice-Evans, C. 1999. Antioxidant activity applying and improved ABTS radical cation decolorization assay. Free Radical Biology \& Medicine 26:1231-1237.

Rice-Evans, AC; Miller, NJ; Paganga, G. 1997. Antioxidant properties of phenolic compounds. Trends in Plant Science 2:152-158.

Rodríguez, JL; Valdés, O; Alemán, A. 2006. Evaluación de la actividad antioxidante de cinco hierbas aromáticas. Ciencia y Tecnología de los Alimentos 16:30-36.

Rodríguez, VMJ; Tomassini, SLR; Mancade Nadra, MC; Strasser de Sad, AM. 2010. Antioxidant capacity and antibacterial activity of phenolic compounds from argentinean herbs infusions. Food Control 21:779-785.

Sala, JM; Lafuente, MT. 2000. Catalasa enzyme activity is related to tolerance of mandarin fruits to chilling. Postharvest Biology and Technology 20:81-89.

Sankat, CK; Maharaj, R. 1997. Papaya. In Mitra, SK. ed. Postharvest physiology and storage of tropical and subtropical fruits. CAB International. Great Britain. p. 167-190.

SAS (Statistical Analysis System). 2002. SAS/STAT users guide: Statics, Ver. 9.0. SAS Institute, Inc. Cary, NC, USA. 1503 p.

Scalzo, J; Politi, A; Pellegreni, N; Mezzeti, B; Battino, M. 2005. Plant genotype affects total antioxidant capacity and phenolic content in fruit. Basic Nutritional Investigation 21:207-213.

Scandalios, JG. 1993. Oxygen stress and superoxide dismutases. Plant Physiology 101:7-12.

Serteser, A; Kargioglu,M; Gök, V; Bagcl, Y; Musa, OM; Arslan, D. 2009. Antioxidant properties for some plants growing wild in Turkey. Grasas y Aceites 60:147-154.

Shivashankara, KS; Isobe, S; Al-Haq, MI; Takenaka, M; Shina, T. 2004. Fruit antioxidant activity, ascorbic acid, total phenol, quercitin, and carotene of Irwin mango fruits stored at low-temperature after high electric field treatment. Journal of Agricultural of Food Chemistry 52:1281-1286.

Stauffer, CE. 1989. Enzyme assays for food scientists. Van Nostrand Reinhold. USA. 317 p.

Tao, F; Zhang, M; Ging, Hang-qing, Y. 2007. Effect of vacuum cooling on physiological changes in the antioxidant system of mushroom under different storage conditions. Journal of Food Engineering 79:1302-1309.

Tavarini, S; Degl'Innocenti, E; Remorini, D; Massai, R; Guidi, L. 2008. Antioxidant capacity, ascorbic acid, total phenols and carotenoids changes during harvest and after storage of Hayward kiwifruit. Food Chemistry 107:282-288. 
Tawaha, K; Alali, FQ; Gharaibeh, M; Mohamed, M; ElElimat, T. 2007. Antioxidant activity and total phenolic content of selected Jordanian plant species. Food Chemistry 104:1372-1378.

Tepe, B; Sokmen, M; Askin, AH; Sokmen, A. 2006. Screening of the antioxidant potential of six Salvia species from Turkey. Food Chemistry 95:200-204.

Thompson, KA. 2003. Fruit and vegetables: harvesting, handling and storage. Blackwell Publishing, Ltd. New York, USA. 482 p.

Trujillo, VBA; Zavaleta, MHA; Mora, HME; López, DHA. 2006. Efecto del $\mathrm{CaCl}_{2}$ sobre la actividad enzimática antioxidante durante la vida florero de gerbera (Gerbera jamesonni $\mathrm{H}$. Bolux Ex Hook F.). Revista Chapingo Serie Horticultura 12:203-209.

Vargas, MA; Camelo, RAP; Cuenca, NCE. 2005. Capacidad antioxidante durante la maduración de arazá (Eugenia stipitata Mc Vaugh). Revista Colombiana de Química 34:57-65.

Velioglu, YS; Mazza, G; Gao, L; Oomah, BD. 1998. Antioxidant activity and total phenolic in selected fruits, vegetables, and grain products. Journal of Agricultural and Food Chemistry 46:4113-4117.
Vijayakumar, R; Zhao, Chang-Xing, Z; Gopal, R; Abdul, CJ. 2009. Non-enzymatic and enzymatic antioxidant variation in tender and mature leaves of Sttrychnos nux-vomica L. (Family: Loganiaceae). Comptes Rendus Biologies 332:52-57.

Viña, SZ; Chaves, AR. 2006. Antioxidant responses in minimally processed celery during refrigerated storage. Food Chemistry 94:68-74.

Vranová, E; Inzé, D; Breusengem Van, F. 2002. Signal transduction during oxidative stress. Journal of Experimental Botany 53:1227-1236.

Waterman, PG; Mole, S. 1994. Analysis of phenolic plant metabolites. Blackwell Scientific Publications, Oxford, UK. 238 p.

Wojdylo, A; Oszmianski, J; Czemerys, R. 2007. Antioxidant activity and phenolic compounds in 32 selected herbs. Food Chemistry 105:940-949.

Yanishlieva, NV; Marinova, E; Pokorny, J. 2006. Natural antioxidant from herbs and spices. European Journal Lipids Science Technology 108:776-793.

Zheng, W; Wang, SY. 2001. Antioxidant activity and phenolic compounds in selected herbs. Journal of Agriculture and Food Chemistry 49:5165-5170. 
